\title{
Melanocitoma del nervio óptico: fisiopatología, consideraciones clínicas y novedades diagnósticas
}

\section{Optic nerve melanocytoma: pathophysiology, clinical considerations, and diagnostic novelties}

\author{
M. Camila Estrada-Villarrea/* \\ Servicio de Oftalmología, Universidad el Bosque, Bogotá, Colombia
}

\section{Resumen}

El melanocitoma del nervio óptico es un tumor raro, benigno, pigmentado, de la papila, que en algunos casos puede causar alteraciones visuales e incluso puede confundirse con una patología maligna del polo posterior como el melanoma. En esta revisión se destacan sus características clínicas y fisiopatológicas, y se realiza una actualización en el tema del diagnóstico con las nuevas tecnologías disponibles, como por ejemplo, la tomografía-angiografía de coherencia óptica.

Palabras clave: Nervio óptico. Melanocitoma. Neoplasia benigna. Fisiopatología. Clínica. Diagnóstico.

\begin{abstract}
Optic nerve melanocytoma is a rare, benign, pigmented tumor of the optic disc that may cause visual complications in few cases and can be easily confused with a malignant melanoma. This review aims to highlight its clinical characteristics and pathophysiology, as well as to update the diagnostic methods available, like optical coherence tomography angiography.
\end{abstract}

Key words: Optic nerve. Melanocytoma. Benign neoplasm. Pathophysiology. Clinic. Diagnostic.

\section{Introducción}

El melanocitoma del nervio óptico es una neoplasia benigna que, debido a sus características, confunde a sus observadores, ya que simula ser una neoplasia maligna con posible compromiso vital del paciente. Anteriormente, los pacientes que presentaban esta neoplasia eran enucleados por sospecha de malignidad y compromiso sistémico.

Gracias a Zimmerman, en $1962^{1-8}$ se documentó una serie de casos enucleados en quienes al realizar el correspondiente estudio histopatológico se descubrió las características benignas del melanocitoma. Esto, asociado a las nuevas tecnologías como fotografía, angiografía y tomografía de coherencia óptica (OCT), ha hecho posible dar un mejor abordaje a estos pacientes y evitar enucleaciones innecesarias, lo que les ha permitido conservar su visión y anatomía. Asimismo, esta condición se ha asociado con complicaciones raras pero que pueden ocurrir, como por ejemplo, alteraciones del campo visual $(40-90 \%)^{9,10}$, crecimiento de la neoplasia $(15 \%)$ y transformación maligna $(1-2 \%)^{10}$. Entre otras, cabe mencionar el edema de la papila
Disponible en internet: 01-05-2020 Rev Mex Oftalmol. 2020;94(3):131-139 www.rmo.com.mx (http://creativecommons.org/licenses/by-nc-nd/4.0/). 
(25\%), compresión vascular (3\%) y exudación retiniana $(12 \%)^{2,4,5}$.

El propósito de esta revisión es identificar información básica y actualizada sobre el tema para resaltar la importancia en su diagnóstico y manejo para su aplicación en el contexto oftalmológico.

\section{Métodos}

Se realizó una revisión de la literatura utilizando las bases de datos PubMed y Web of Science. En la base de datos de PubMed (MEDLINE) se utilizó una estrategia de búsqueda con términos (MeSH) usando los operadores booleanos AND y OR de la siguiente manera: («Optic Nerve Neoplasms»[Mesh] OR «Optic Nerve Diseases»[Mesh]) AND «Melanocytes»[Mesh]. Como resultado se obtuvieron 13 artículos. La búsqueda en Web of Science se llevó a cabo con la estrategia de búsqueda: Optic Nerve AND Melanocytoma. Como resultado se obtuvieron 50 artículos. La palabra "Melanocitoma» no existe como término Mesh, por lo que se usaron otros términos para tratar de englobar a este último y lograr completar la exploración. Se obtuvo como resultado un total de 63 artículos. No se revisó la literatura gris ni se usaron filtros de limitación, como fecha de publicación. Solo se seleccionaron artículos en inglés y en español, por lo que se eliminaron 9 artículos por esta razón. Al realizar la lectura de los abstracts se eliminaron 10 artículos más, ya que no correspondían a la temática a tratar en esta revisión. Se incluyó un capítulo de un libro y un artículo tomado de las referencias bibliográficas de uno de los artículos seleccionados y se eliminaron los artículos repetidos. Con lo que quedó una revisión total de 45 documentos.

\section{Epidemiología}

La edad media de diagnóstico es a los 50 años, con un rango entre 1 y 91 años según la revisión de Shields ${ }^{9-14}$. Sin embargo, en otros reportes se encuentra un rango entre los 17 y los 74 años $^{14}$. Un hallazgo similar se encontró en un estudio en población coreana con una edad media de diagnóstico de 46 años ${ }^{15}$.

El melanocitoma forma parte del $0.55 \%$ de los tumores intraoculares pigmentados, y su localización intraocular más frecuente es el nervio óptico ${ }^{16}$. Existen reportes con incidencia de más casos en mujeres (62\%) que en hombres $(38 \%)^{14,17}$, datos que son correspondientes en población coreana $(63 \%)^{15}$ y europea $(62 \%)^{7}$.

Se ha encontrado igual incidencia entre razas ${ }^{9}$, sin embargo, otros reportes indican mayor frecuencia en personas con piel densamente pigmentada y caucási$\cos ^{4,14}$. Shields, et al., en un estudio de 115 pacientes, encontraron que esta lesión se presentaba en el $65 \%$ de personas de raza blanca, el $29 \%$ de personas de raza negra y el $7 \%$ entre indios (1\%), árabes (1\%), asiáticos $(3 \%)$ e hispanos $(2 \%)^{2,10,18}$. La mayoría de las veces es unilateral ${ }^{9}\left(99 \%\right.$ de los pacientes $\left.{ }^{10}\right)$, y afecta más al ojo derecho (56\%) que al izquierdo $(43 \%)^{2}$.

Debido a la ausencia o baja incidencia de síntomas, su diagnóstico usualmente es en un control oftalmológico de rutina.

Un estudio en población europea publicado por el Dr. Zografos encontró síntomas visuales en el $26 \%$ de los pacientes ${ }^{7}$. Del mismo modo, en población coreana, los síntomas visuales estuvieron presentes en un $22 \%$ de los pacientes ${ }^{15}$, y en un $26 \%$ de los pacientes estadounidenses evaluados por Shields $s^{9,10,19}$.

A pesar de ser una lesión benigna, su contraparte, el melanoma, es una condición fatal. La malignización del melanocitoma es rara, sin embargo, se ha docu-

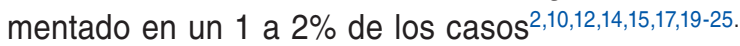

\section{Presentación clínica}

El melanocitoma del nervio óptico en la mayoría de casos es asintomático, es por ello que su diagnóstico principalmente se da en una valoración oftalmológica de rutina ${ }^{3,23,24}$, sin embargo, existen reportes de síntomas presentados por los pacientes con esta afección. La disminución de la agudeza visual se ha encontrado hasta en el $26 \%$ de los pacientes ${ }^{9,10,19}$, que han tenido compromiso isquémico de la arteria central de la retina o necrosis del tumor $7,9,10,22,26-28$, no obstante, también se ha descrito que la pérdida de visión puede ser secundaria a un edema axonal por compresión del nervio $3,7,21,22,27,29$. Se describió un caso con ceguera secundaria a compresión por melanocitoma en un niño de 12 años, a pesar de este reporte, una pérdida profunda de la visión es rara ${ }^{30}$.

Aparte de la disminución de la visión, se han descrito otros síntomas, por ejemplo, Shields, en su estudio de 115 casos encontró que entre los síntomas presentados por los pacientes había: visión borrosa (16\%), destellos de luz (4\%), miodesopsias (4\%) y sin síntomas $(76 \%)^{2}$.

Se ha documentado la presencia de un defecto pupilar aferente relativo (DPAR) en el $30 \%$ de los ojos con melanocitoma, incluso sin afección de la agudeza visual ${ }^{19,31}$. A pesar de este hallazgo, Shields hace referencia al $9 \%$ de los pacientes en uno de sus estudios².

Estos síntomas, al parecer, son secundarios a pequeñas compresiones en las fibras nerviosas o al tamaño 
del tumor, lo que también llevó a cuestionarse su compromiso en el campo visual, y se encontró que hasta el $90 \%{ }^{9}$ de los pacientes con DPAR tenían algún tipo de defecto.

La mejor forma de observar el tumor y sus características es por medio de la oftalmoscopia. En cuanto a la coloración del tumor, se sabe que es una lesión que varía entre el café oscuro y el negro ${ }^{26,31}$. Particularmente, en el estudio de Shields tuvieron un $97 \%$ de casos de color negro y solo un $3 \%$ de color cafée,10.

Su localización en el disco óptico suele ser inferotemporal ${ }^{11,15,24,32,33}$. Puede centrarse únicamente en el nervio óptico en el $15 \%$ de los casos, sin embargo, en el $54 \%$ puede comprometer la coroides adyacente y en el $30 \%$, la retina neurosensorial ${ }^{9,10,19}$. En cuanto al área comprometida, Shields encontró que, en el $75 \%$ de los casos, el 50\% o menos del área del disco está comprometida, mientras que en el $12 \%$ compromete más del $90 \%{ }^{4}$.

Con relación a la forma, los márgenes del disco tienden a ser de tipo fibrilado o plumoso $0^{4,16,19,24}$.

Estas lesiones se han visto asociadas a melanocitosis ocular en el $8 \%$ de los pacientes ${ }^{2,9}$, y se han encontrado otras asociaciones oculares en pacientes con melanocitoma, sin embargo, no existe evidencia científica que compruebe su relación, como por ejemplo, hipoplasia del nervio óptico $(2 \%)$, retinitis pigmentosa (1\%) e hipertrofia congénita del epitelio pigmentario de la retina $(E P R)^{2}$. También se ha descrito la presencia de un nevo coroideo concomitante con melanocitoma hasta en el 47 a $50 \%$ de los pacientes ${ }^{3,4,13,33}$.

Se reportaron dos casos indicando la asociación de melanocitoma con la membrana neovascular coroidea peripapilar, que causaba disminución de la visión y ponía en duda la benignidad de la lesión. Los criterios que permitieron a los cirujanos un abordaje crítico del caso fueron la estabilidad del tamaño del tumor por angiografía y ultrasonografía, así como la ausencia de otras características que hicieran sospechar maligni$\mathrm{dad}^{27}$. En el primer caso decidieron dar manejo quirúrgico a la membrana, con mejoría de la agudeza visual ${ }^{27}$. En el segundo caso existía presencia de membrana neovascular coroidea con edema macular que se trató con bevacizumab con buenos resultados visuales ${ }^{12}$. La incidencia de esta patología se ha descrito en el $1 \%$ de los pacientes con melanocitoma ${ }^{12,34}$.

Entre otras asociaciones, se han reportado hallazgos típicos de vasculopatía polipoide coroidea en un paciente con melanocitoma del nervio óptico ${ }^{18}$, que se presenta como lesiones subretinianas redondeadas pequeñas de color rojo-naranja, en relación con el nervio óptico ${ }^{35}$, sin embargo, no es el único caso reportado en la literatura. Específicamente el doctor Rouvas describe la asociación y su posible tratamiento con terapia fotodinámica + aflibercept intravítreo con buenos resultados ${ }^{18}$.

Por otro lado, algunas investigaciones han encontrado la presencia concomitante de melanocitoma del nervio óptico con tumores de células derivadas de la cresta neural tipo meningioma. No obstante, al ser reportes de caso, no se conocen las implicaciones, por lo tanto, solo se puede afirmar que un meningioma puede estar presente de forma concomitante en un paciente con melanocitoma ${ }^{36}$.

Kaliaperumal, et al. encontraron el vínculo entre hipertensión arterial (HTA) y melanocitoma fundamentados en el origen de las células pigmentarias, las células de la médula adrenal y los feocromocitos, ya que todas provienen de la cresta neural ${ }^{11}$. Estos investigadores encontraron HTA asociada a melanocitoma del disco óptico y metabolitos hormonales en orina ${ }^{11}$.

Con respecto a lo anterior, en Corea, un $19 \%$ de los pacientes se asociaron a HTA y un $8 \%$ a tumores coexistentes derivados de la cresta neural ${ }^{15}$.

Con relación a algunas enfermedades sistémicas, el melanocitoma se ha encontrado en condiciones como la neurofibromatosis tipo $2^{2,9}$, carcinoma de células basales ${ }^{9}$, vitíligo ${ }^{9}$, facomatosis pigmentovascularis ${ }^{10} \mathrm{y}$ meningioma intracraneal ${ }^{9,10}$, aunque al existir solo algunos reportes de caso, su relación aún no se ha podido confirmar.

Para concluir las asociaciones encontradas, un extraño caso mostró la presencia de fosfenos inducidos por sonidos fuertes, predominantemente en la oscuridad, en el ojo con melanocitoma de un paciente. Se cree que esta relación tiene un componente más central, al presentarse persistencia de los fosfenos luego de la enucleación ${ }^{29}$.

Entre algunas complicaciones asociadas a la presencia del melanocitoma, hay estudios que han evidenciado edema del disco $(25 \%)^{19}$, palidez del disco $(2 \%)^{2,10}$, edema intrarretiniano $(16 \%)^{19,26}$, fluido subretiniano $(14 \%)^{19,26}$, exudados intrarretinianos $(12 \%)^{19,26}$, neovascularización retiniana/coroidea $(1 \%)^{2,10}$, hemorragias focales $(5 \%)^{19}$. Estas últimas usualmente son leves, y se cree que son secundarias a vascularización yuxtapapilar.

Se han reportado siembras vítreas en un $4 \%^{2,9,19}$, en ocasiones estas siembras se extienden a la cámara anterior y forman un pseudohipopion negro, además, en estos pacientes se producen necrosis isquémicas del tumor con compromiso severo de la visión. 
Las oclusiones vasculares se reportan en un $3 \%$ de los $\operatorname{casos}^{19,26}$, divididas así: arteria central de la retina $(2 \%)$ y de rama $(1 \%)^{2}$. Por lo tanto, son causa de disminución considerable de la agudeza visual. Se describió, en una paciente australiana, el crecimiento y transformación maligna de un melanocitoma, que causó oclusión vascular y glaucoma neovascular secundario, que asociado al dolor y, más que nada, al efecto de masa produjo bradicardia sintomática por un reflejo oculocardíaco ${ }^{17}$.

Se ha reportado también, en la literatura, la presencia de neurorretinitis secundaria a necrosis e inflamación ${ }^{8}$.

Hay estudios que han demostrado que estas lesiones son estables, con un promedio de tamaño basal de $2 \mathrm{~mm}(1-10 \mathrm{~mm})$ y grosor de $1 \mathrm{~mm}(0,5-3 \mathrm{~mm})$ para población americana ${ }^{2,10}$, y de $3.1 \mathrm{~mm}(1.6-4.4 \mathrm{~mm})$ y $1.9 \mathrm{~mm}(0.8-2.4 \mathrm{~mm})$, respectivamente, para población coreana $^{15}$.

El crecimiento de lesiones benignas confirmadas por patología postenucleación se ha documentado en un 10 a $15 \%$ de los casos $9-13,16,17,21-24,30,31,33,37,38$, luego de varios años de seguimiento, aproximadamente de 5 a 20 años ${ }^{4}$.

Por lo tanto, un crecimiento mínimo no debe confundirse con transformación maligna. Asimismo, una disminución del tamaño se ha evidenciado en un 3\% de los casos ${ }^{4}$.

La malignización, aunque rara, también es posible, $y$ se ha evidenciado en el 1 a $2 \%$ 12,14,15,17,19-23,25,34. Algunas de las características que pueden hacer sospechar una transformación maligna son, por ejemplo, una lesión pequeña que inicialmente solo comprometa el disco óptico, que presente crecimiento y extensión con pérdida de la visión secundaria a una oclusión vascular $^{9,10}$. Otra característica sospechosa es el grosor mayor a $1.5 \mathrm{~mm}$ en el momento del diagnóstico, que a su vez es un factor de riesgo para crecimiento del tumor ${ }^{10,15,19}$. Dentro de los reportes de caso en donde se ha documentado la malignización, se habla de lesiones al inicio planas $(2 \mathrm{~mm})$, con crecimiento en altura de 4 y $6.7 \mathrm{~mm}$ en un periodo de 5 y 6 años de seguimiento, respectivamente ${ }^{3}$. Por lo que, en el reporte del Dr. Sharma, en India, pudieron sospechar una lesión maligna que contaba con todas las características de un melanocitoma a excepción de una altura en ecografía de $5.2 \mathrm{~mm}^{3}$.

Por lo que se refiere a estudios genéticos, recientemente se ha demostrado una asociación genética en la mutación de GNAQ/11 en varias lesiones de tipo pigmentado, como en el nevo azul, melanosis ocular, melanocitoma ciliocoroideo, melanoma y lesiones en piel.
Otro estudio confirma su asociación con melanocitoma del nervio óptico y del iris ${ }^{6}$. Por otro lado, las mutaciones en BAP1 están presentes hasta en el $84 \%$ de los melanomas uveales metastásicos, y en este estudio sugieren confirmar con estudios de mayor muestra las implicaciones de un BAP1 en un paciente con melanocitoma, ya que su presencia podría implicar tratamientos agresivos en pacientes con lesiones aparentemente benignas ${ }^{6}$.

\section{Patología}

En los análisis histopatológicos se han descrito dos tipos de células:

- Células tipo 1: Son células de forma oval o redondea$\mathrm{da}$, con abundante pigmento citoplasmático intenso ubicado en grandes melanosomas, de radio núcleo-citoplasma bajo, tamaño uniforme de las células y el núcleo. Organelos y nucleolos prominentes escasos. Las características de estas células son similares a las de los pacientes con melanocitosis ${ }^{14,16,27,29,39}$.

- Células tipo 2: Tienen forma de huso, son menos pigmentadas, con melanosomas pequeños en forma de bastón, de radio núcleo-citoplasma alto, con nucleolos más prominentes y mayor número de organelos citoplasmáticos 9 ,14,16,27,39.

Así mismo se pueden encontrar macrófagos llenos de pigmento flotando en el vítreo que pueden causar glaucoma secundario ${ }^{14}$.

Un estudio realizó la correlación histopatológica del tumor evidenciando edema del tejido neural prelaminar, desplazamiento retiniano y pliegues. Las capas comprimidas también se vieron como pliegues en la patología 5 .

\section{Fisiopatología}

Se piensa que es una lesión congénita, tipo hamartoma que se desarrolla de melanocitos aberrantes de la lámina coroidalis ${ }^{3,11,23,32,39,40}$, sin embargo, es raro encontrarla en niños. Shields reportó un caso, en el que se documentó la aparición de una lesión pigmentada del nervio óptico en un paciente con reportes fotográficos previamente normales, lo que sugiere una aparición de novo en la edad adulta o una pigmentación de una lesión congénita amelanótica ${ }^{9,10,40}$.

\section{Diagnóstico}

El diagnóstico de estas lesiones se realiza típicamente por oftalmoscopia, sin embargo, suelen ser necesarios ciertos paraclínicos como las fotografías, 


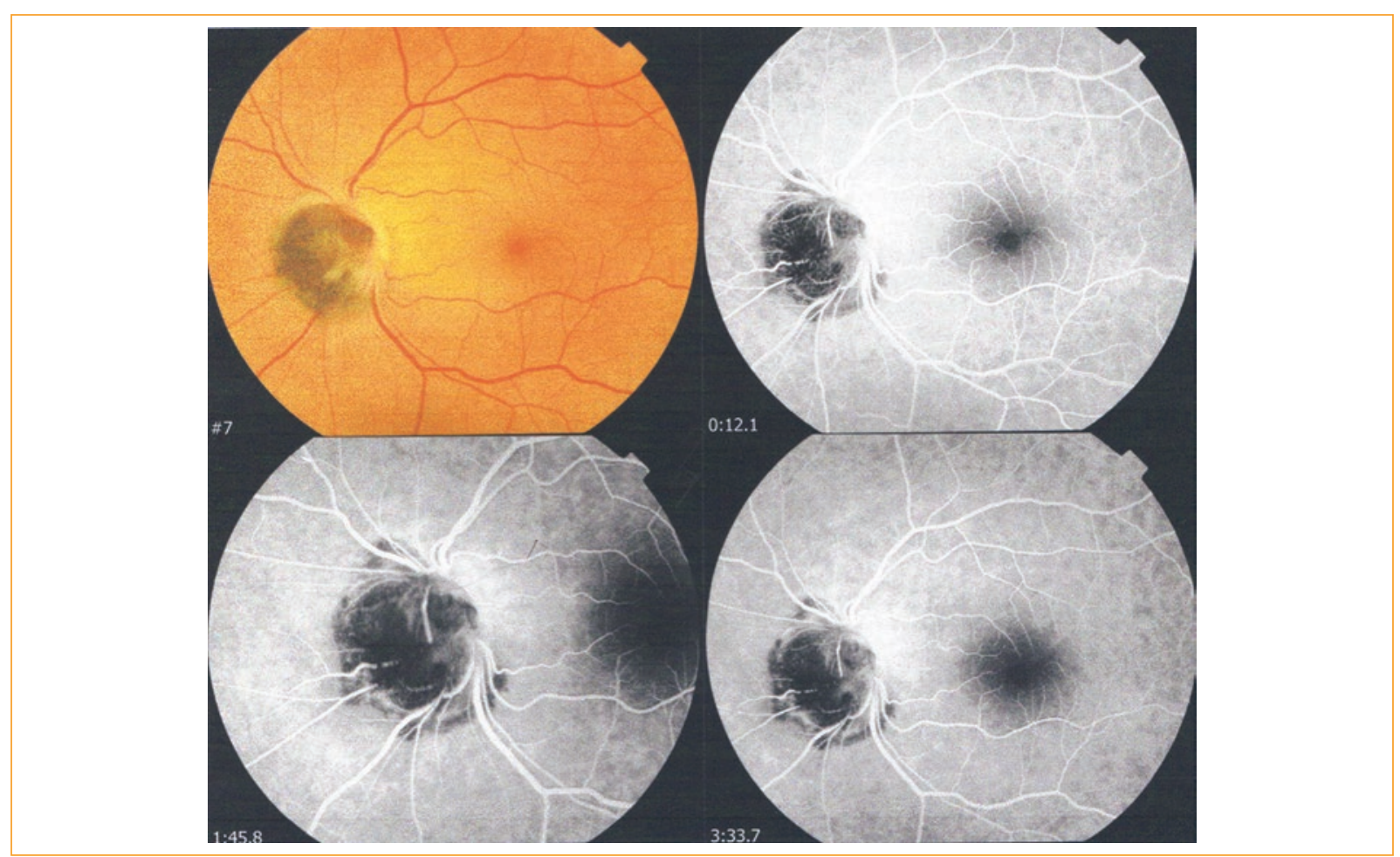

Figura 1. Superior izquierda: Corresponde a la foto a color de un melanocitoma del nervio óptico. Superior derecha: Angiografía fluoresceínica. Inferior Izquierda: Fase temprana. Inferior derecha: Fase tardía. En esta foto se evidencia compromiso del disco óptico por la lesión pigmentada, y el bloqueo en la fluorescencia secundario a la densa pigmentación (fotografía cortesía de la Dra. Zeiad Eldaly, de Eldaly, et al. ${ }^{23}$ ).

angiografía fluoresceínica, OCT y ecografía como parte del apoyo diagnóstico de las posibles complicaciones y síntomas asociados a la lesión, así como también para documentar su estabilidad y crecimiento. Shields sugiere seguimiento fotográfico de las lesiones cada 1 a 2 años ${ }^{2,4}$ y Sharma, el seguimiento del tamaño de la lesión por ecografía ${ }^{3}$.

\section{Angiografía retinofluoresceínica}

En cuanto a la angiografía retinofluoresceínica, se evidencia hipofluorescencia y autohipofluorescencia 4,19,21,34,38, probablemente secundarias a las células densamente pigmentadas y compactadas ${ }^{19,38}$, con relativa poca vascularización y ausencia de lipofuscina ${ }^{38}$. La retina adyacente en la autofluorescencia es isoautofluorescente ${ }^{41}$. La región adyacente del tumor puede verse hiperfluorescente cuando existe edema asociado del disco ${ }^{4,19}$ o fluido subretiniano ${ }^{2}$ (Fig. 1). Esto coincide también con los hallazgos en la angiografía con verde de indocianina, donde esta lesión se muestra hipofluorescente ${ }^{10,21}$.

En términos de fluorescencia, en China se realizó un estudio con autofluorescencia con luz infrarroja, que parte del principio de ser hiperfluorescente en lugares con alta concentración de melanina como la coroides, especialmente en la fóvea, y muestra así una hiperfluorescencia del melanocitoma que permite resaltar y delinear de forma adecuada la lesión ${ }^{38}$.

\section{Ultrasonografía ocular}

Una lesión de más de $0.5 \mathrm{~mm}$ de elevación puede ser detectada por una ecografía ocular o una tomografía axial computarizada, sin embargo, este examen no puede diferenciar un melanocitoma de otro tipo de lesiones elevadas ni la extensión microscópica del tumor $^{9,10,19,38}$. Los hallazgos típicos de un melanocitoma en ecografía modo B son solidez acústica y

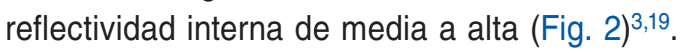

\section{Tomografía de coherencia óptica}

Es posible también la realización de una OCT, sin embargo, será útil únicamente evaluando las condiciones asociadas, como el fluido subretiniano y el edema retiniano cistoide ${ }^{9,20,38,41}$, así como el grosor retiniano sobre 

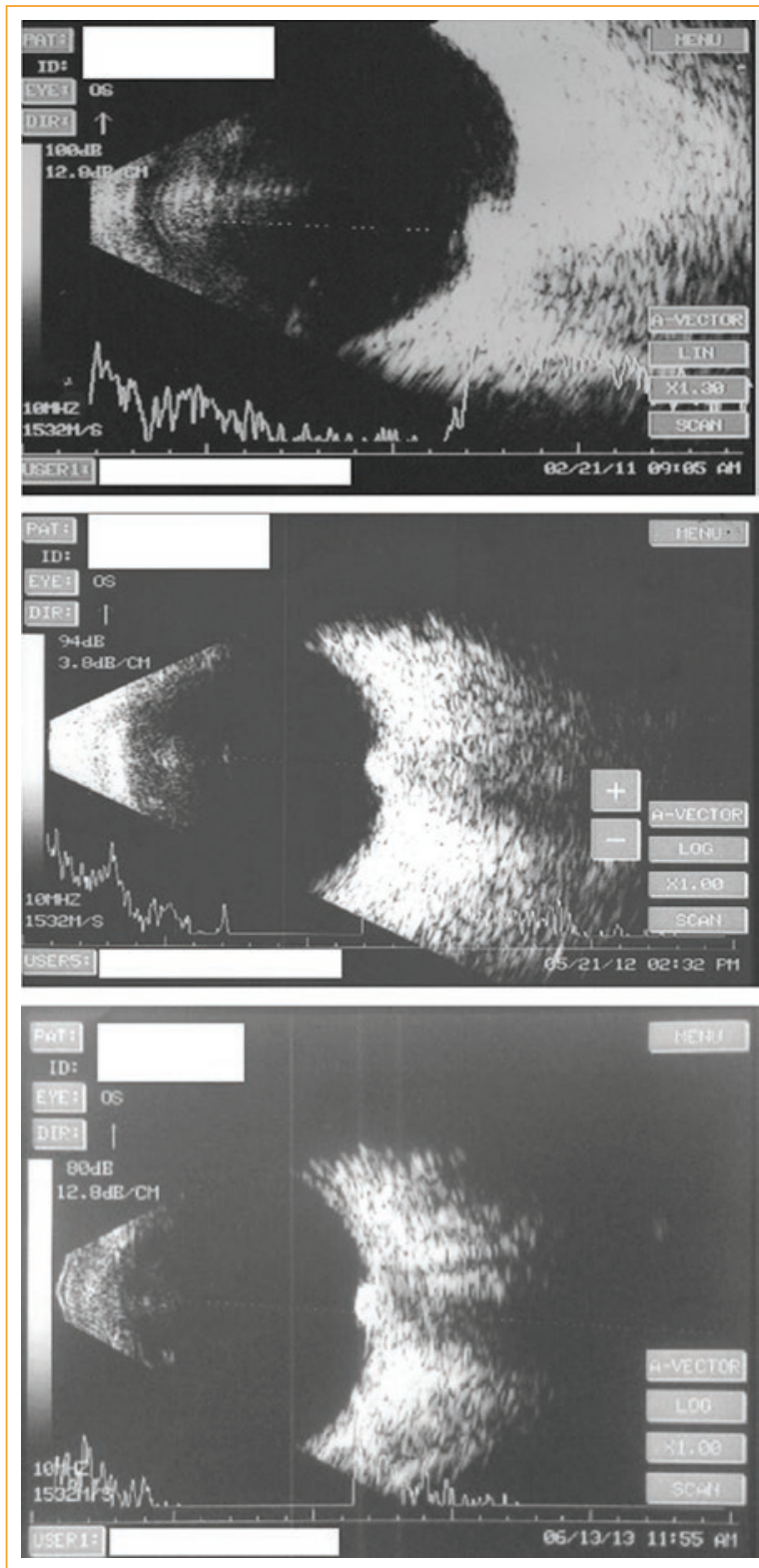

Figura 2. Fotografías seriadas de una ultrasonografía modo B del ojo izquierdo de un paciente con melanocitoma del nervio óptico en 2011 (superior), 2012 (central) y 2013 (inferior). Evidencia que no hubo cambios en el tamaño de la masa, ecogenicidad o excavación coroidea (fotografía cortesía de la Dra. Zeiad Eldaly, de Eldaly, et al. ${ }^{23}$ ).

el tumor ${ }^{20}$, ya que la densidad del tumor bloquea el paso de la luz y evita observar otros detalles internos.

Entre técnicas de spectral domain (SD) o time domain (TD), el SD muestra ventajas sobre el TD, es más rápido y tiene mayor resolución ${ }^{32}$. Es posible observar una masa prepapilar, lisa y en forma de domo de alta reflectividad que es continua con la capa de

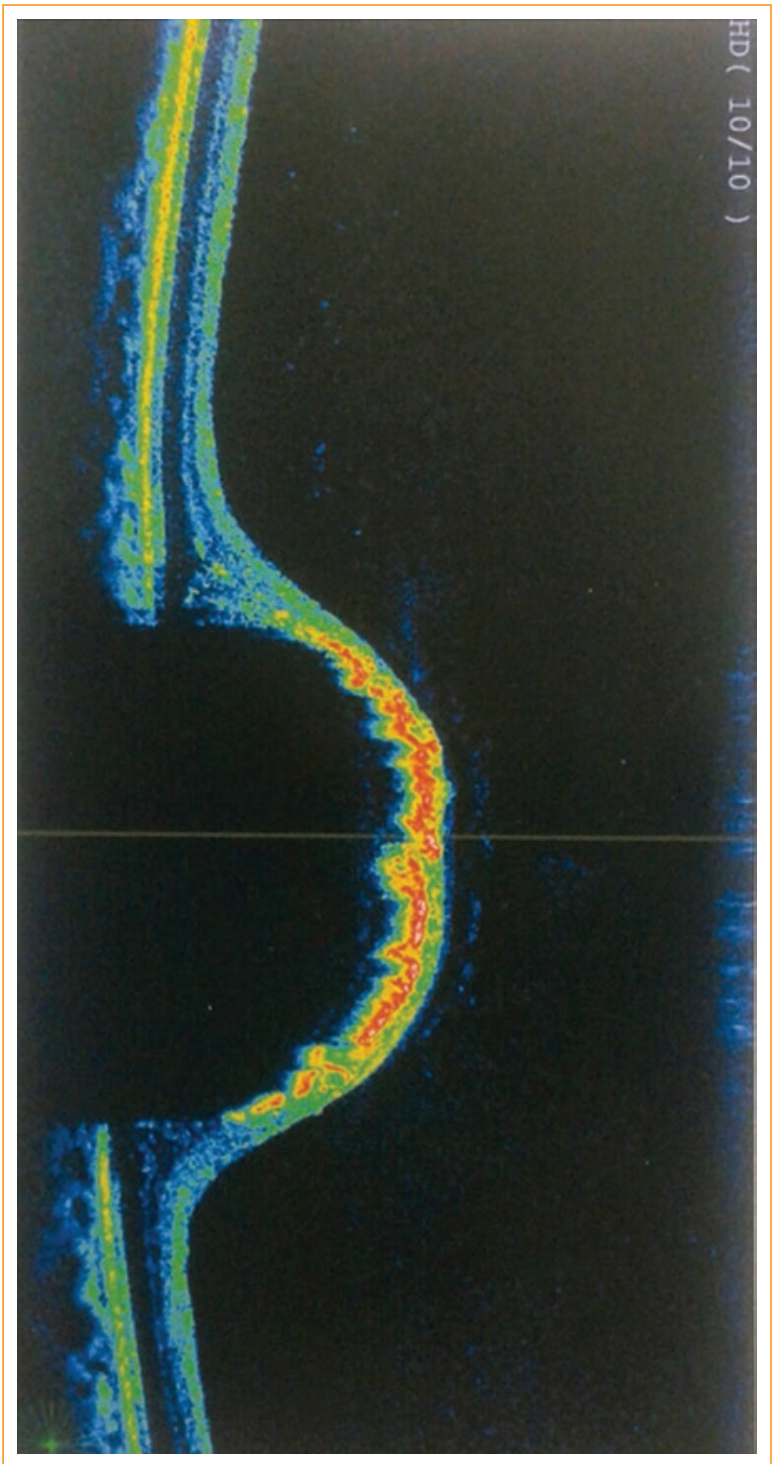

Figura 3. Tomografía óptica coherente de la cabeza del nervio óptico. Evidencia una superficie altamente reflectiva con sombra óptica de las estructuras posteriores a la lesión (fotografía cortesía de la Dra. Zeiad Eldaly, de Eldaly, et al. ${ }^{23}$ ).

fibras nerviosas adyacente $5,19,26,32,38,39$, y que genera una densa sombra acústica $5^{5,10,19,26,32,38,39}$ que representa el vacío interno de la masa sin otros detalles (Fig. 3) ${ }^{5,26}$. Al realizar una correlación histopatológica, la superficie hiperreflectiva corresponde a un área de degeneración gliótica e hiperpigmentación ${ }^{5}$. En algunos casos también es posible documentar las siembras vítreas $^{10}$, sobre todo cuando se combina la OCT con la oftalmoscopia láser de barrido ${ }^{5}$.

Se realizó un estudio en Japón donde usaron el SD-OCT para identificar características en los vasos 

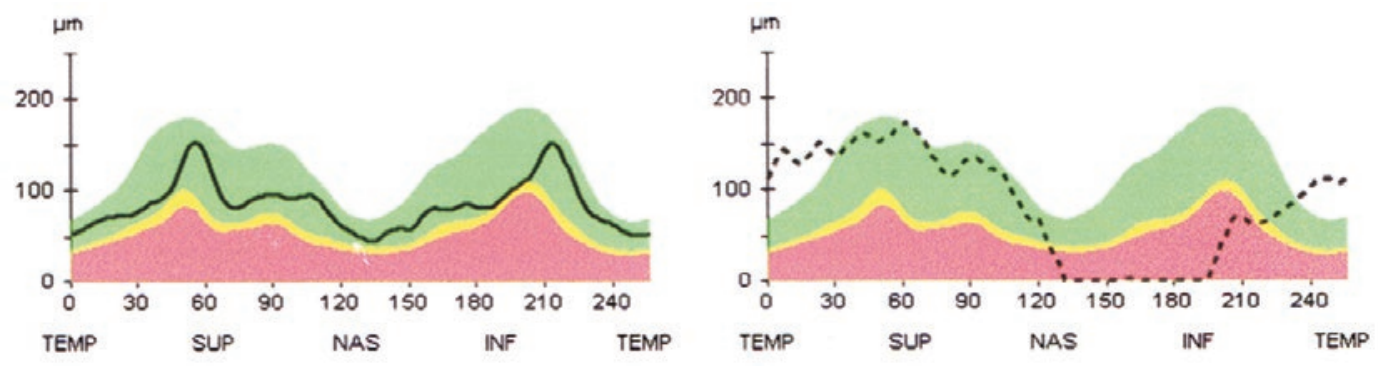

Figura 4. Mapa de grosor de capa de fibras nerviosas (ojo derecho e izquierdo, respectivamente). Se puede apreciar una configuración normal en patrón de doble colina en el ojo derecho y una pérdida de capa de fibras nerviosas correspondiente a la localización de la lesión en el ojo izquierdo (fotografía cortesía de la Dra. Zeiad Eldaly, de Eldaly, et al.23). TEMP: cuadrante temporal; SUP: cuadrante superior; NAS: cuadrante nasal; INF: cuadrante inferior.

retinianos de personas con melanocitoma del disco óptico, y se observaron ciertas estructuras tubulares sobre la superficie apical y retiniana del tumor, que correspondieron a las primeras ramas de la arteria y vena central de la retina, lo que se presume genera el componente irregular de las superficies. Los vasos más profundos son oscurecidos por la sombra acústica, y estos típicamente mostraban ciertos puntos hiperreflectivos perivasculares de distintos tamaños a diferencia de las ramas principales y los vasos en las porciones donde no había tumor ${ }^{20}$.

Estos vasos, además, mostraban un curso tortuoso desde la superficie del tumor hacia la retina, lo que puede ser asociado a las complicaciones vasculares del tumor $^{20}$. Los puntos hiperreflectivos perivasculares observados pueden corresponder a extravasaciones de lípidos y proteínas desde el tumor, melanófagos o células tumorales ${ }^{20}$. Un estudio evaluó la presencia de drusens enterradas en el disco óptico en asociación a melanocitoma, que por su ausencia de calcificación no son visibles por otras técnicas, pero sí por medio de $\mathrm{OCT}^{42}$.

\section{Tomografía-angiografía óptica coherente}

Con angio-OCT (OCT-A), la red capilar radial peripapilar resalta sobre la masa en casos en los que no existe compromiso retiniano. Con esta técnica puede no ser posible ver los vasos profundos ${ }^{10}$. Sin embargo, a diferencia de la angiografía, permite ver capas vasculares más profundas, las cuales no es posible evaluar por ese método por la densa pigmentación ${ }^{21}$.

Es importante mencionar que la OCT-A no se influencia por la pigmentación al basarse en el movimiento de los eritrocitos para realizar la toma. En un reporte de caso de un paciente con melanocitoma estable, se detectaron cambios en el campo visual sugestivos de glaucoma, la OCT-A permitió identificar disminución en el flujo vascular en las áreas afectadas del disco correspondientes a los defectos en el campo visual, lo que sugiere una etiología glaucomatosa y no compresiva del defecto ${ }^{21}$. La OCT-A es un método diagnóstico prometedor al no tener las complicaciones invasivas de la angiografía, además puede evaluar capas vasculares profundas, la vascularización del tumor y la capa de fibras nerviosas por separado ${ }^{19,21,43}$, sin embargo, hacen falta estudios, ya que hasta el momento solo existen reportes de casos en la literatura que respaldan esta hipótesis.

\section{Campo visual}

En el campo visual, se han observado alteraciones en un $40 \%^{10}$ a $90 \%^{9,23,34}$ de los pacientes. Comprendiendo defectos como, por ejemplo, aumento mínimo y mayor de la mancha ciega ${ }^{4}$ en el 15 y $75 \%$ de los pacientes, respectivamente ${ }^{4], 9,16}$, escalones nasales en el $10 \%{ }^{9}$, y defectos relativos y arqueados absolutos del haz de fibras nerviosas en el $20 \%$ en cada caso $^{4,9}$. Shields en su estudio encontró que el $24 \%$ de sus pacientes tenían campos alterados, el $32 \%$ con aumento de la mancha ciega y el $24 \%$ con un defecto en uno de los cuadrantes (Fig. 4$)^{2}$.

\section{Resonancia magnética}

La resonancia magnética puede detectar progresiones macroscópicas en la porción retrolaminar del nervio cuando se usa concomitantemente con gadolinio, sin embargo, no detecta progresiones microscópicas $^{5,26}$. La resonancia es también crucial para 
descartar lesiones retroorbitarias y tumores malignos como el melanoma ${ }^{22}$. No distingue bien entre un melanoma y un melanocitoma, ya que ambos son hiperintensos en $\mathrm{T} 1$ e hipointensos en $\mathrm{T} 2^{19}$.

\section{Potenciales visuales evocados}

En India reportaron un caso clínico de un paciente a quien se le realizó potenciales visuales evocados, y que mostró resultados alterados por afección de la agudeza visual, con un retraso en la conducción del nervio óptico ${ }^{3}$.

\section{Diagnóstico diferencial}

Dentro de las lesiones que se debe tener en cuenta a la hora de valorar un paciente con sospecha de melanocitoma, encontramos las siguientes: melanocitoma yuxtapapilar de la coroides, nevus coroideo, hiperplasia del EPR, hamartoma combinado de la retina y EPR, adenoma del EPR, melanoma metastásico al nervio óptico, hemorragia vítrea epipapilar, melanoma coroideo, Pits del nervio óptico, entre otros $24-26,34,37,40$.

\section{Tratamiento}

Dada su condición benigna, no es necesario realizar manejo alguno de la lesión. La documentación fotográfica anual de la lesión y la evaluación fundoscópica, así como la indagación de posibles síntomas que sugieran progresión son suficientes en estos casos ${ }^{9,10}$. Existen casos en los que puede ser necesario realizar una biopsia con aguja fina y su consiguiente enucleación en caso de demostrarse malignidad por las características en la biopsia o la expresión genética ${ }^{10,14,24,28}$. El tratamiento de condiciones asociadas al melanocitoma como membranas epirretinianas, edema macular o glaucoma puede ser necesario para conservar o mejorar la agudeza visual, así como el manejo de sus posibles complicaciones.

\section{Responsabilidades éticas}

Protección de personas y animales. Los autores declaran que para esta investigación no se han realizado experimentos en seres humanos ni en animales.

Confidencialidad de los datos. Los autores declaran que han seguido los protocolos de su centro de trabajo sobre la publicación de datos de pacientes.

Derecho a la privacidad y consentimiento informado. Los autores han obtenido el consentimiento informado de los pacientes y/o sujetos referidos en el artículo. Este documento obra en poder del autor de correspondencia.

\section{Conflicto de intereses}

Se declara no tener conflictos de intereses.

\section{Agradecimientos}

A la Dra. Diana Rey, por su colaboración en la realización de este proyecto.

A la Dra. Zeiad Eldaly, por la contribución de las fotografías aquí presentadas.

\section{Bibliografía}

1. Zimmerman L, Garron LK. Melanocytoma of the optic disk. Int Ophthalmol Clin. 1962;2:431-40.

2. Shields JA, Shields CL, Demirci H, Mashayekhi A. Melanocytoma of optic disc in 115 cases: The 2004 Samuel Johnson Memorial Lecture, part 1. Ophthalmology. 2004;111(9):1739-6.

3. Sharma PM, Sangal K, Malik P, Mathur MB. Malignant Transformation of Optic Disc Melanocytoma? A Clinical Dilemma at Presentation with a Review of the Literature. Ophthalmologica 2002;216(4):292-5.

4. Brown GC, Shields JA. Tumors of the Optic Nerve Head. Surv Ophthalmol. 1985;29(4):239-64.

5. Finger PT, Natesh S, Milman T. Optical Coherence Tomography: Pathology Correlation of Optic Disc Melanocytoma. Ophthalmology. 2010;117(1):114-9.

6. Francis JH, Wiesner T, Milman T, Won HH, Lin A, Lee V, et al. Investigation of Somatic GNAQ, GNA11, BAP1 and SF3B1 Mutations in Ophthalmic Melanocytomas. Ocular Oncology and Pathology. 2016;2(3):171-7.

7. Zografos L, Othenin-Girard CB, Desjardins L, Schalenbourg A, Chamot L, Uffer S. Melanocytomas of the optic disk. Am J Ophthalmol. 2004;138(6):964-9.

8. Shanmugam MP, Khetan V, Sinha P. Optic Disk Melanocytoma With Neurorretinitis. Retina. 2004;24(2):317-8.

9. Shields JA, Shields CL, Demirci H, Mashayekhi A, Eagle RC. Melanocytoma of the Optic Disk: A Review. Surv Ophthalmol. 2006;51(2):93-104.

10. Shields CL, Joeffe L, Shields JA. Melanocitoma of the Optic Disc. En: Schachat AP, Wilkinson CP, Hinton DR, Sadda SR, Wiedemann Peter, eds. Ryan's Retina. $6^{\text {th }}$. ed. London: Remedica Medical Education and Publishing; 2018.

11. Kaliaperumal S, Gupta A, Nongrum B, Rao VA, Srinivasan R. Case Reports of Three Patients Showing Optic Nerve Head Melanocytoma and Systemic Hypertension. Ophthalmologica. 2006;221(1):62-4.

12. Urrets-Zavalia JA, Crim N, Esposito E, Correa L, Gonzalez-Castellanos ME, Martinez D. Bevacizumab for the treatment of a complicated posterior melanocytoma. Clinical Ophthal. 2015;9:455-9.

13. Costa J, Vargas E, Mendonça AP, Barbosa MS. Growing optic disc melanocytoma: what to do? Neuro-Ophthalmology. 2002;27(1-3):39-43.

14. Saro F, Clua A, Esteva E, Carreras A, Adán A, Lerma E. Cytologic diagnosis of ocular melanocytoma: a case report. Acta Cytologica. 2008;52(1):87-90.

15. Lee CS, Bae JH, Jeon IH, Byeon SH, Koh HJ, Lee SC. Melanocytoma of the optic disk in the Korean population. Retina. 2010;30(10):1714-20.

16. Al-Hinai A, Edelstein C, Burnier MN. Unusual case of melanocytoma. Canadian J Ophthalmol. 2004;39(4):461-3

17. Salinas LR, Cesar M. Malignant Transformation of Optic Nerve Melanocytoma into Melanoma Associated with Ocular Ischemic Syndrome and Oculocardiac Reflex: Case Report and Review of the Literature. Semin Ophthalmol. 2017;32(2):253-6.

18. Rouvas A, Gouliopoulos NS, Moschos MM, Theodossiadis P. Optic disk melanocytoma associated with polypoidal choroidal vasculopathy lesions, after combination treatment of photodynamic therapy and intavitreal aflibercept (Eylea), a case report. BMC Ophthalmol. 2018;18(267):2-6.

19. Elias EK, John E, Jose GPK. Optic disc melanocytoma- a rare case. J Evolution Med Dent Sci. 2018;7(14):1818-21.

20. Okubo A, Unoki K, Yoshikawa H, Ishibashi T, Sameshima M, Sakamoto T. Hyperreflective dots surrounding the central retinal artery and vein in optic disc melanocytoma revealed by spectral domain optical coherence tomography. Jpn J Ophthalmol 2013;57(1):108-12. 
21. Kita Y, Holló G, Murai A, Kita R, Hirakata A. Optical coherence tomography angiography findings of an optic disc melanocytoma in a glaucoma eye. Int Ophthalmol. 2019;39(3):677-82

22. Mohindra S, Mohindra S, Dhawan A, Saikia U, Mankoo RS. Primary malignant melanoma of the optic nerve sheath: a case report. Surgical Neurology. 2009;71(3):372-5.

23. Eldaly H, Eldaly Z. Melanocytoma of the Optic Nerve Head, Thirty-Month Follow-Up. Semin Ophthalmol. 2015;30(5-6):464-9.

24. de Alba MA, Villegas VM, Gold AS, Wildner A, Ehlies FJ, Latiff A, et al. Clinical findings and genetic expression profiling of three pigmented lesions of the optic nerve. Case reports in ophthalmological medicine 2015;2015:1-7

25. Salvanos P, Utheim TP, Moe MC, Eide N, Bragadóttir R. Autofluorescence imaging in the differential diagnosis of optic disc melanocytoma. Acta Ophthalmologica. 2015:93(5):476-80.

26. Shields CL, Perez B, Benavides R, Materin MA, Shields JA. Optical coherence tomography of optic disk melanocytoma in 15 cases. Retina. 2008;28(3):441-6.

27. Tran H, Bovey E, Uffer S, Zografos L. Peripapillary choroidal neovascularization associated with melanocytoma of the optic disc: a clinicopathologic case report. Graefe's Arch Clin Exp Ophthalmol. 2006:244(10):1367-9.

28. Shields JA, Shields CL, Eagle RC, Singh AD, Berrocal MH, Berrocal JA. Central Retinal Vascular Obstruction Secondary to Melanocytoma of the Optic Disc. Arch Ophthalmol. 2001;119(1):129-33.

29. Kim IK, Dryja TP, Lessell S, Gragoudas E. Melanocytoma of the Optic Nerve Associated With Sound-Induced Phosphenes. Archives of Ophthalmology 2006;124(2):273-6.

30. Shields JA, Shields CL, Ehya H, Dhaliwal RS. Total Blindness From Presumed Optic Nerve Melanocytoma. Ame J Ophthalmol. 2005; 139(6):1113-4.

31. Shields JA. Tumors and pseudotumors of the optic disc. Acta Ophthalmologica Scandinavica. 2000:78(2):156-63.

32. Shah VA, Vincent RD, Desai K, Gallimore G, Rupani M. Documentation of optic disc melanocytoma by spectral and time domain optical coherence tomography. Can J Ophthalmol. 2009;44(5):603-4.
33. Alkatan $\mathrm{HM}, \mathrm{Al}-$ Rashaed $\mathrm{S}$. Choroidal melanoma in association with juxtapapillary melanocytoma. Can J Ophthalmol. 2008;43(2):250-1.

34. Reshma Rodriguez G, Mendoca TM, Pai SG, Amruthavalli KS. Optic Disc Melanocytoma Associated with Choroidal Neovascular Membrane: An Unusual Presentation. J Clin Diagnostic Res. 2018; 12(11):NDO-ND02.

35. Bartlett HM, Willoughby B, Mandava N. Polypoidal Choroidal Vasculopathy in a Patient With Melanocytoma of the Optic Nerve. Retina. 2001; 21(4):396-9.

36. Shinoda K, Hayasaka S, Nagaki Y, Kadoi C, Kurimoto M, Okada E. Melanocytoma of the left optic nerve head and right retrobulbar optic neuropathy compressed by a tuberculum sellae meningioma. Ophthalmologica. 2000;214(2):161-3.

37. Puri P, Prasad S, Rennie IG. Organized vitreous hemorrhage masquerading as an optic disc melanocytoma. Europ J Ophthalmol. 2003; 13(2):215-7.

38. Zhang P, Hui YN, Xu WQ, Zhang ZF, Wang HY, Sun DJ, et al. Infrared autofluorescence, short-wave autofluorescence and spectral-domain optical coherence tomography of optic disk melanocytomas. Int J Ophthalmol. 2016;9(5):713-6.

39. Chaudhary R, Arora R, Mehta DK, Singh M. Optical Coherence Tomography Study of Optic Disc Melanocytoma. Ophthalmic Surg Lasers Imaging Retina. 2006;37(1):58-61.

40. Shields JA, Shields CL, Piccone M, Snady-McCoy LC. Spontaneous appearance of an optic disk melanocytoma in an adult. Ame J Ophthalmol. 2002;134(4):614-5.

41. Guerra RL, Marback EF, Silva IS, Maia Junior Ode O, Marback RL. Autofluorescence and spectral-domain optical coherence tomography of optic disk melanocytoma. Arq Bras Oftalmol. 2014;77(6):400-2.

42. Lee K, Hwang J, Woo S. Optic Disc Drusen Associated with Optic Nerve Tumors. Optom Vis Sci. 2015;92(4S):S6-S75.

43. Carnevali A, Querques L, Zucchiatti I, Scorcia V, Bandello F, Querques G. Optical Coherence Tomography Angiography Features in Melanocytoma of the Optic Nerve. Ophthalmic Surg Lasers Imaging Retina. 2017:48(4):364-6. 